\title{
Graphic User Interface for Reliable and Repeatable Quantification of Neuron Morphology and Microstructural Analysis
}

\author{
Jeffrey La ${ }^{1}$, Briana Mason², Tiffany Donaldson², and Chandra Yelleswarapu ${ }^{1}$ \\ 1. Physics Department, University of Massachusetts Boston, Boston Massachusetts, USA. \\ 2. Psychology Department, University of Massachusetts Boston, Boston, Massachusetts, USA.
}

Steroid hormones influence anxiety-like behavior and interact with the prototypical anti-anxiety medication, diazepam (Valium) in humans and in animals [1]. In Long Evans female rats, the proestrus stage of the estrous cycle (when estradiol and progesterone levels peak) increases anxiety-like behavior as well as improvements in diazepam efficacy [2]. Progesterone and diazepam modulate the $\gamma-$ aminobutyric acid (GABA) system, which is implicated in anxiety disorders and important in neural development. Thus GABA plays a major inhibitory role in mammalian cortex. Additionally, estrous changes also relate to fluctuations in brain-derived neurotrophin factor (BDNF), with BDNF expression or efflux peaking and falling with rising estradiol and declining progesterone, respectively. Fluctuations in BDNF are also implicated in anxiety-like behavior in animals and the pathogenesis and treatment of anxiety disorders in humans [3]. Given this previous hormonal, pharmacokinetic and morphological data implicating elevated responses to amphetamine and more pronounced stimulant sensitization in females, there is a need to examine any acute changes in terms of stained neuron count, size, eccentricity, aspect ratio, and count density in medial prefrontal cortex GABAergic neuron levels.

To carry out quantitative analysis, it is common practice to perform the analysis manually because the notion of determining what is and is not significant in images of new research (and therefore inherently low data sets) has yet to be algorithmically solved. This process not only introduces systematic and hard-to-characterize biases, but it is extremely time intensive. Through interdisciplinary collaboration, we developed an open source user-friendly graphic user interface (GUI) in MATLAB from the ground up shown in figure 1, for neuron reconstruction and microstructural analysis that addresses unmet demands posed by current problems in biomedical research- capturing dynamic changes in neurons and objectively quantifying morphology differences at a cost-effective level.

Fixed brain post-mortem coronal cross-sections were mounted on to motorized Zeiss AxioObserver inverted microscope that is equipped with a CCD camera (AxioCam HRm3) and connected to a desktop computer, to capture tiled images in a Z-stack. User selected brain regions are then imaged using a 40x objective (EC Plan-Neofluar 40x/0.75) in a tiling pattern with 5\% overlap. Each brain region was then focus stacked, stitched in a batch process via ImageJ macros, and fed into the custom GUI developed in MATLAB, as shown in figure 1. Within the GUI, we stitch images using a powerful, optimizes version of phase correction [4]. Otsu's method and maximum entropy thresholding are used to separate the background and physical thresholds such as minimum and maximum size, eccentricity, circularity of neuron are used to determine what is and is not significant in the image. Resultant images are then used to quantify BDNF and PV-positive immunoreactive images in high and low anxiety rats. We counted immunoreactive cells within each of the regions of interest (ROI) within a $500 \mu \mathrm{m} \times 500 \mu \mathrm{m}$ reticle, using every other serial section for a total of 6 sections per animal per slide, 5-6 animals per group. Each ROI was fed into the GUI for repeatable quantification of stained structures by recording the user's parameters and outputting metrics such as stained structure's area, perimeter, circularity, eccentricity, relative stain intensity and its standard deviation, which all serve as potential filter parameters. 
Figure 1 displays cell count and average cell body size/area obtained using aforementioned GUI. The results suggest greater female BDNF-positive neurons in the central amygdala (CeA), medial prefrontal cortex (mPFC) and nucleus accumbens (NAc) that parallel large average soma sizes in these same regions for females. This indicates sex-dependent interactions with diazepam treatment and as a whole, the data relates differences in BDNF protein levels and PV-containing GABA neurons in sex disparity in anxiety-like behavior and response to diazepam. Although additional work is needed to provide direct evidence of the link between GABA density, BDNF protein, and estrogen and/or progesterone levels, this GUI has helped the experimentalist non-programmer to reliable and repeatable metrics traditionally unfeasible.

\section{References:}

[1] J. Simpson, et al, "Sex differences in baseline and drug-induced behavioural responses in classical behavioural tests," Progress in Neuro-Psychopharmacology \& Biological Psychiatry 37 (2012) 227-236. [2] C. A. Frye, S. M. Petralia, M. E. Rhodes, "Estrous cycle and sex difference in performance on anxiety tasks coincide with increases in hippocampal progesterone and $3 \alpha, 5 \alpha$-THP,” Pharmacology Biochemistry Behavior 67 (2000) 587-596.

[3] L. S. Cohen, et al, "Treatment of mood disorders during pregnancy and postpartum depression," Psychiatric Clinics of North America 33 (2010), 273-93.

[4] S. Preibisch, S. Saalfield, and P. Tomancak, "Globally optimal stitching of tiled 3D microscopic image acquisitions,” Bioinformatics 25 (2009) 1463-1465.
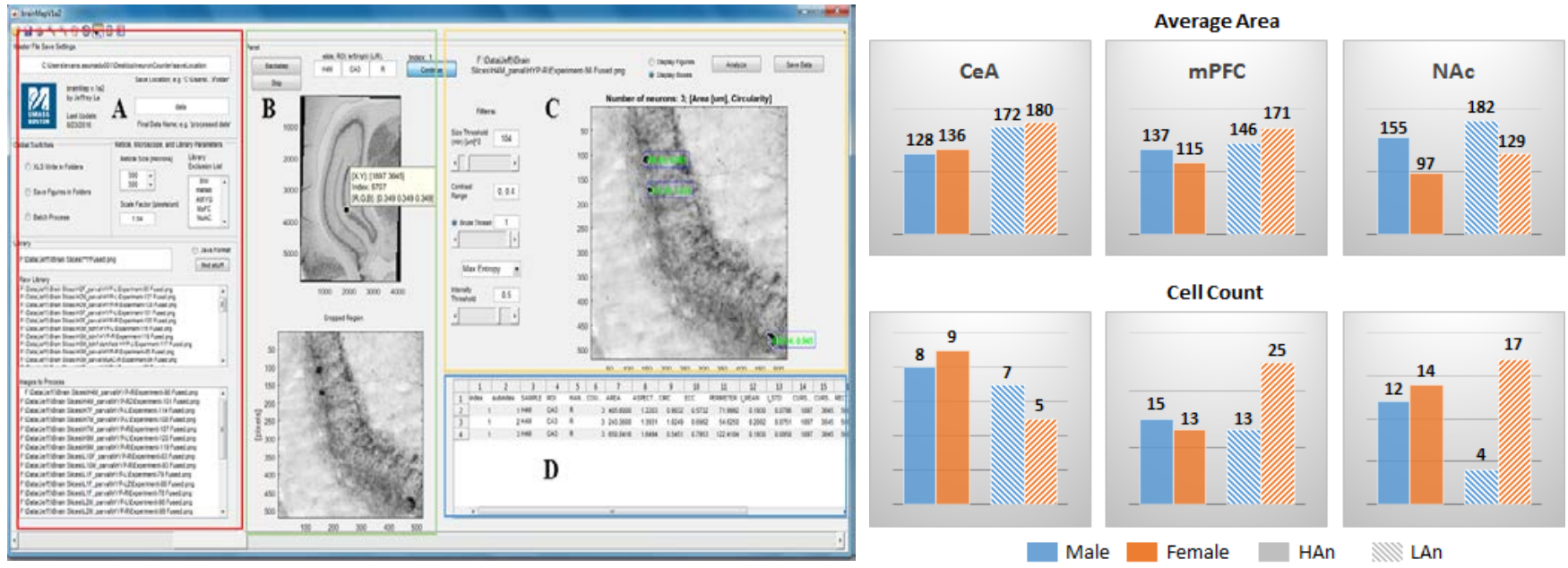

Figure 1. Screenshot of the MATLAB GUI for neuron quantification (left) and the resulting BDNF stained neuron data between high (HAn) and low (LAn) anxiety, male and female rats in the central amygdala (CeA), medial prefrontal cortex (mPFC), and nucleus accumbens (NAc) (right). Panel A contains file read and data save locations, reticle size and scale factor selection windows, and the image name filter list. The user chooses the images to be processed from the list of found images in the library. In Panel B, the user toggles through the library and chooses the region of interest, displayed on the bottom. In Panel C, the user adjusts filter parameters as needed and refreshes morphological calculations via the "Analyze" button on the top right. When the user is satisfied, the "Save Data" button outputs the data into a *.csv. Panel D previews the data output, which includes image list index, stained object index, sample name, region of interest location, handedness, and quantiative metrics. 